\title{
Radial Distribution of the Center of Gravity of Random Points on a Unit Circle
}

\author{
F. Scheid ${ }^{2}$ \\ This paper describes some Monte Carlo computations carried out on the Standards \\ Electronic Automatic Computer (SEAC) which are related to the problem of random walks.
}

In 1905 Pearson $[1]^{3}$ suggested the problem of a random walk involving $n$ steps of equal length and arbitrary direction in the plane. In 1906 Kluyver [2] obtained the solution

$$
P(r, n)=r \int_{0}^{\infty}\left[J_{0}(x)\right]^{n} J_{1}(r x) d x
$$

giving the probability of being no farther than $r$ from the starting point after $n$ steps of unit length. 'This integral has been calculated by Greenwood and Durand [3] for $n=6(1) 24$, and $r=0.5(.5) n$, using punched-card tables of the Bessel functions. These tables were not extensive enough to handle the values $n=3,4$, and 5 . For $n=2$ one finds by a simple argument, $P(r, 2)=(2 / \pi) \arcsin (r / 2)$.

The problem described in the title is essentially the same as Pearson's. Its solution for $n=3,4$, and 5

\footnotetext{
1 Preparation of this paper was initiated while the author was a member of the Numerical Analysis Training Program held at the National Bureau of Standards and sponsored by the National Science Foundation, 1957.

and sponsored by the National Science Foundation, 195
2 Present address: Boston University, Boston, Mass.

${ }^{3}$ Figures in brackets indicate the literature references at the end of this paper.
}

has been obtained to roughly three decimal places by a direct sampling procedure, using SEAC. A set of $n$ pseudorandom numbers is generated by using the multiplication method of Taussky and Todd [4]. A linear transformation converts these into a sample from the uniform distribution over $(-\pi, \pi)$. The center of gravity is found and classified in one of the intervals $j / 32 \leq R<(j+1) / 32, \quad j=0, \ldots ., 31$. A new sample is then taken. Both frequency table and cumulative distribution are printed. Computation of Kluyver's integral for these values of $n$ is possible but lengthy. If the integrand is machine computed as needed, many hours of computer time are consumed because of the two independent parameters $r$ and $n$. For limited accuracy the sampling approach seems better. The distributions for $n=2$ and 6 have also been obtained for accuracy checks. Using a theorem of Kolmogoroff [5], a brief calculation indicates that, for $n=2$, the probability of a maximum error less than or equal to 0.01 after 6,000 samples have been taken is approximately one-half. A glance at table 1 shows that in fact the

TABLE 1 .

\begin{tabular}{|c|c|c|c|c|c|c|c|c|c|c|c|}
\hline \multirow[t]{2}{*}{$32 R$} & \multirow[b]{2}{*}{ Freq } & \multirow{2}{*}{$\begin{array}{c}n=2 \\
\text { Cum }\end{array}$} & \multirow[b]{2}{*}{ Exact } & \multirow{2}{*}{\multicolumn{2}{|c|}{ Freq ${ }^{n=3}$ Cum }} & \multicolumn{2}{|c|}{$n=4$} & \multicolumn{2}{|c|}{$n=5$} & \multicolumn{2}{|c|}{$n=6$} \\
\hline & & & & & & Freq & Cum & Freq & Cum & Cum & $G-D$ \\
\hline 1 & 121 & .0197 & .0199 & 7 & .001 & 36 & .005 & 28 & .004 & .0000 & .0000 \\
\hline 2 & 133 & .0413 & .0398 & 37 & .007 & 87 & .018 & 80 & .016 & & \\
\hline 3 & 126 & .0618 & .0598 & 58 & .017 & 128 & .038 & 158 & .040 & & \\
\hline 4 & 124 & .0820 & .0798 & 67 & .028 & 169 & .063 & 185 & .068 & & \\
\hline 5 & 129 & .1030 & 0999 & 95 & .043 & 209 & .094 & 210 & .099 & & \\
\hline 6 & 111 & .1211 & .1201 & 113 & .061 & 192 & .123 & 308 & .146 & & \\
\hline 7 & 123 & .1411 & .1404 & 141 & .084 & 266 & .163 & 381 & .203 & & \\
\hline 8 & 115 & .1598 & .1609 & 172 & .112 & 289 & .207 & 361 & .257 & .2910 & .2932 \\
\hline 9 & 129 & .1808 & .1816 & 224 & .149 & 238 & .242 & 382 & .314 & & \\
\hline 10 & 142 & .2039 & .2023 & 336 & .203 & 316 & .290 & 350 & .367 & & \\
\hline 11 & 123 & .2240 & .2234 & 466 & .279 & 335 & .340 & 361 & .421 & & \\
\hline 12 & 138 & .2464 & .2447 & 344 & .335 & 360 & .394 & 384 & .479 & & \\
\hline 13 & 126 & .2669 & .2663 & 291 & .383 & 357 & .448 & 358 & .533 & & \\
\hline 14 & 157 & .2925 & .2883 & 285 & .429 & 365 & .503 & 384 & .590 & & \\
\hline 15 & 126 & .3130 & .3106 & 269 & .473 & 365 & .558 & 374 & .647 & & \\
\hline 16 & 125 & .3333 & .3333 & 255 & .514 & 405 & .618 & 330 & .696 & .7665 & .7672 \\
\hline 17 & 150 & .3577 & .3565 & 223 & .551 & 353 & .672 & 317 & .744 & & \\
\hline 18 & 158 & .3835 & .3803 & 189 & .581 & 255 & .710 & 324 & .802 & & \\
\hline 19 & 135 & .4054 & .4047 & 208 & .615 & 275 & .751 & 273 & .834 & & \\
\hline 20 & 148 & .4295 & .4298 & 185 & .645 & 262 & .790 & 224 & .867 & & \\
\hline 21 & 157 & .4551 & .4558 & 215 & .680 & 182 & .818 & 179 & .894 & & \\
\hline 22 & 158 & .4808 & .4826 & 197 & .712 & 159 & .842 & 143 & .916 & & \\
\hline 23 & 173 & .5090 & .5106 & 183 & .742 & 163 & .866 & 131 & .935 & & \\
\hline 24 & 190 & .5399 & .5399 & 201 & .775 & 168 & .892 & 109 & .952 & .9749 & .9732 \\
\hline 25 & 191 & .5710 & .5708 & 188 & .805 & 167 & .917 & 93 & .966 & & \\
\hline 26 & 211 & .6053 & .6038 & 183 & .835 & 131 & .936 & 70 & .967 & & \\
\hline 27 & 197 & .6374 & 6393 & 163 & .862 & 102 & .952 & 45 & .983 & & \\
\hline 28 & 247 & .6776 & .6783 & 176 & .890 & 87 & .965 & 39 & .989 & & \\
\hline 29 & 262 & .7202 & .7221 & 170 & .918 & 87 & .978 & 36 & .994 & & \\
\hline 30 & 308 & .7703 & .7737 & 162 & .944 & 76 & .989 & 24 & .998 & & \\
\hline 31 & 424 & .8394 & .8407 & 163 & .971 & 45 & .996 & 12 & .999 & & \\
\hline 32 & 987 & 1.0000 & 1. 0000 & 178 & 1.000 & 27 & 1.000 & 3 & 1.000 & 1.0000 & 1.0000 \\
\hline Number of samples & 6144 & & & 6144 & & 6656 & & 6656 & & & \\
\hline
\end{tabular}


TABLE 2 .

\begin{tabular}{|c|c|c|c|c|c|c|}
\hline $32 R$ & \multicolumn{2}{|c|}{$n=3$} & \multicolumn{2}{|c|}{$n=4$} & \multicolumn{2}{|c|}{$n=5$} \\
\hline $\begin{array}{l}1 \\
2 \\
3 \\
4 \\
5 \\
6 \\
7 \\
8\end{array}$ & $\begin{array}{r}4 \\
10 \\
17 \\
19 \\
48 \\
45 \\
76 \\
92\end{array}$ & $\begin{array}{l}.001 \\
.003 \\
.006 \\
.010 \\
.019 \\
.028 \\
.043 \\
.061\end{array}$ & $\begin{array}{r}9 \\
29 \\
50 \\
59 \\
71 \\
88 \\
69 \\
109\end{array}$ & $\begin{array}{l}.002 \\
.007 \\
.017 \\
.029 \\
.043 \\
.060 \\
.073 \\
.095\end{array}$ & $\begin{array}{r}7 \\
17 \\
21 \\
40 \\
61 \\
72 \\
99 \\
113\end{array}$ & $\begin{array}{l}.002 \\
.006 \\
.011 \\
.021 \\
.036 \\
.053 \\
.077 \\
.105\end{array}$ \\
\hline $\begin{array}{l}9 \\
10 \\
11 \\
13 \\
14 \\
15 \\
16\end{array}$ & $\begin{array}{l}107 \\
160 \\
289 \\
203 \\
203 \\
176 \\
177 \\
185\end{array}$ & $\begin{array}{l}.082 \\
.113 \\
.169 \\
.209 \\
.249 \\
.283 \\
.318 \\
.354\end{array}$ & $\begin{array}{l}133 \\
126 \\
151 \\
191 \\
221 \\
231 \\
232 \\
287\end{array}$ & $\begin{array}{l}.121 \\
.145 \\
.175 \\
.212 \\
.255 \\
.300 \\
.346 \\
.402\end{array}$ & $\begin{array}{l}106 \\
120 \\
142 \\
149 \\
177 \\
167 \\
227 \\
217\end{array}$ & $\begin{array}{l}.131 \\
.160 \\
.195 \\
.231 \\
.274 \\
.215 \\
.371 \\
.424\end{array}$ \\
\hline $\begin{array}{l}17 \\
18 \\
19 \\
20 \\
21 \\
22 \\
23 \\
24\end{array}$ & $\begin{array}{l}170 \\
172 \\
176 \\
196 \\
185 \\
196 \\
196 \\
172\end{array}$ & $\begin{array}{l}.387 \\
.421 \\
.455 \\
.493 \\
.529 \\
.568 \\
.606 \\
.639\end{array}$ & $\begin{array}{l}248 \\
237 \\
235 \\
235 \\
215 \\
191 \\
229 \\
210\end{array}$ & $\begin{array}{l}.450 \\
.496 \\
.542 \\
.588 \\
.630 \\
.667 \\
.712 \\
.753\end{array}$ & $\begin{array}{l}242 \\
234 \\
237 \\
207 \\
208 \\
178 \\
179 \\
168\end{array}$ & $\begin{array}{l}.483 \\
.540 \\
.598 \\
.648 \\
.699 \\
.742 \\
.786 \\
.827\end{array}$ \\
\hline $\begin{array}{l}25 \\
26 \\
27 \\
28 \\
29 \\
30 \\
31 \\
32\end{array}$ & $\begin{array}{l}184 \\
224 \\
220 \\
236 \\
225 \\
239 \\
239 \\
279\end{array}$ & $\begin{array}{r}.675 \\
.719 \\
.762 \\
.808 \\
.852 \\
.899 \\
.946 \\
1.000\end{array}$ & $\begin{array}{r}205 \\
183 \\
188 \\
183 \\
167 \\
152 \\
120 \\
66\end{array}$ & $\begin{array}{r}.793 \\
.829 \\
.866 \\
.901 \\
.934 \\
.964 \\
.987 \\
1.000\end{array}$ & $\begin{array}{r}148 \\
156 \\
117 \\
97 \\
77 \\
65 \\
34 \\
14\end{array}$ & $\begin{array}{r}.863 \\
.901 \\
.930 \\
.954 \\
.972 \\
.988 \\
.997 \\
1.000\end{array}$ \\
\hline Number of samples.- & 5120 & & 5120 & & 4096 & \\
\hline
\end{tabular}

Washington, September 16, 1957. maximum error is 0.004 for this distribution. Since the inverse square root law is operative, to obtain four decimal-place accuracy would require perhaps two-thousand times as many samples, or a year of SEAC time.

Parallel results are given in table 2 for samples drawn from a triangular distribution over $(-\pi, \pi)$. The pseudorandom numbers are modified by a direct method [6] to produce the required sample.

The question how best to compute a distribution function is not, of course, answered in this paper. It is suggested, however, that even in cases where the solution can be written explicitly, circumstances may make computation by direct sampling preferable.

\section{References}

[1] K. Pearson, The problem of the random walk, Nature (1905).

[2] J. C. Kluyver, A local probability problem, Nederl. Akad. Wetensch., Proc. (1906).

[3] Greenwood and Durand, The distribution of length and components of the sum of $\mathrm{n}$ random unit vectors, Ann. Math. Statistics (1955).

[4] O. Taussky and J. Todd, Generation and testing of pseudo-random numbers, Symposium on Monte Carlo Methods (Univ. of Florida, 1954).

[5] W. Feller, On the Kolmogorov-Smirnov limit theorems for empirical distributions, Ann. Math. Statistics (1948).

[6] J. von Neumann, Various techniques used in connection with random digits, NBS AMS 12 (1951). 